\title{
Unveiling the role of chemical and electronic structure in plasmon-assisted homolysis of alkoxyamines
}

Daria Votkina ${ }^{1}$, Pavel Petunin ${ }^{1}$, Elena Miliutina ${ }^{2}$, Andrii Trelin², Oleksiy Lyutakov², Vaclav Svorcik², Gérard Audran ${ }^{3}$, Jeffrey Havot ${ }^{3}$, Rashid Valiev ${ }^{1,5}$, Lenara I. Valiulina, ${ }^{4}$ Jean-Patrick Joly ${ }^{3}$, Olga Guselnikova $^{1,2} *$ Sylvain R. A. Marque ${ }^{3 *}$ and Pavel Postnikov ${ }^{1,2 *}$

${ }^{1}$ Research School of Chemistry and Applied Biomedical Sciences, Tomsk Polytechnic University, Russian Federation

${ }^{2}$ Department of Solid-State Engineering, University of Chemistry and Technology, Prague, Czech Republic ${ }^{3}$ Aix-Marseille Univ, CNRS, ICR case 551, Avenue Escadrille Normandie-Niemen, 13397 Marseille Cedex 20, France

${ }^{4}$ Tomsk State University, 36, Lenin Avenue, 634050 Tomsk, Russia

${ }^{5}$ University of Helsinki, Department of Chemistry, P.O. Box 55, (A.I. Virtanens plats 1), FIN-00014 University of Helsinki, Finland

\begin{abstract}
The excitation of localized plasmon resonance on nanoparticles followed by the interaction with organic molecules leads to new pathways of chemical reactions. Although a number of physical factors (temperature, illumination regime, type of nanoparticles, etc.) are affecting this process, the role of the chemical factors is underestimated. Challenging this assumption, here we studied the kinetic of plasmoninduced homolysis of five alkoxyamines (AAs) with different chemical and electronic structures using electron paramagnetic resonance (EPR). The kinetic data revealed the dependence of plasmonic homolysis rate constant $\left(k_{\mathrm{d}}\right)$ with the HOMO energy of AAs, which cannot be described by the kinetic parameters
\end{abstract}


derived from thermal homolysis experiments. The observed trend in $k_{\mathrm{d}}$ allowed to suggest the key role of intramolecular excitation mechanism supported by the TDFDT calculations, additional spectroscopic characterization, and control experiments. Our work sheds light on the role of the electronic structure of organic molecules in plasmonic chemistry.

Keywords: plasmon, homolysis, alkoxyamines, plasmon-driven reaction, photocatalysis 


\section{Introduction}

Recently we learned that chemical transformation could be driven using the energy of plasmon resonance excitation as an alternative to classical catalytic approaches ${ }^{1,2}$. Plasmon-active substrates (Au, $\mathrm{Ag}, \mathrm{Cu}, \mathrm{Pt}$ ) are illuminated with a light source leading to the energy generation transformed to the energy required for the activation of molecules. Originally, the plasmonic approach was successfully applied toward enhancing catalysis of inorganic reactions such as $\mathrm{CO} / \mathrm{CO}_{2}$ reduction or water splitting ${ }^{3,4}$, but organic reactions could not shot out for a long 5 . The utilization of plasmon catalysis opens a huge potential for improved selectivity ${ }^{6,7}$, enhanced reaction rates $^{8,9}$, and milder reaction conditions ${ }^{10}$. Up to now, the range of plasmon-assisted organic reactions was reported, such as oxidation of alcohols, amines, and unsaturated compounds; reductive transformations; $\mathrm{C}-\mathrm{C}$ and $\mathrm{C}-\mathrm{N}$ bond formation; and polymerization ${ }^{5}$. Despite the diversity of reactions, mechanisms and fundamental principles ${ }^{11,12}$ are investigated using model reactions such as dimerization of $p$-amino/nitro thiophenol.

The mechanism of interaction between plasmon and organic molecules is still disputable. Nowadays, three main hypotheses have been proposed: (1) reaction acceleration through plasmonic heating, (2) transfer of «hot» carriers to the organic molecule, followed by the formation and relaxation of the excited state; (3) intramolecular excitation of an electron to the LUMO via the decay of the optically excited surface plasmon (SP). ${ }^{13,14}$ All these processes, benefited from plasmonic metal nanostructures, can impel plasmonic chemistry. However, recent studies ${ }^{13,14,15}$ including ours ${ }^{8,10}$ cast a doubt on mechanisms (1) and (2) but provide more evidence about intramolecular excitation process (3) in some cases.

The intramolecular excitation mechanism was also adapted for the hybrid plasmonic substrates to manipulate the energy of excited states via the hybridization between the electronic structures of metals/semiconductors with the molecular orbitals of organic regents. ${ }^{16,17}$ However, none of the theories is considering in detail the effects of the electronic structure of organic molecules on the processes of plasmoninduced transformations. In our recent study using alkoxyamines as a perfect thermal probe for plasmon catalysis (due to 1 st order, one component reaction), we unveiled the effect of the nature of the organic 
molecule on the plasmonic kinetic. We have demonstrated that alkoxyamines (AAs) $\mathrm{R}_{1} \mathrm{R}_{2} \mathrm{NOR}_{3}$ are molecules that homolyze into nitroxides $\left(\mathrm{R}_{1} \mathrm{R}_{2} \mathrm{NO} \bullet\right)$ and reactive alkyl radicals $\left(\mathrm{R}_{3} \bullet\right)$, can be monitored by precise EPR measurements, under plasmon excitation on the gold surface. ${ }^{10,18}$ Thus, there is a genuine need, from both fundamental and applied viewpoints, to elucidate the role of the nature/structure of molecules and their electronic structure to the plasmonic chemistry. Filling this gap promises to unlock an unprecedented control over kinetic and thermodynamic parameters of organic reactions and to carefully develop the plasmonic catalysts for organic transformations.

Here, using the advantages of AAs, we performed a thorough study on the kinetic of the plasmoninduced homolysis of alkoxyamines with the different chemical and electronic structures. We used spherical gold nanoparticles coated by organic protective and stabilizing layer to prevent the direct interaction between gold surface with alkoxyamines. The analysis of the reaction rates allows us to prove the importance of the electronic features of organic molecules in plasmon-assisted transformations.

Results of previous investigation of the plasmonic homolysis of AAs and some other molecules 9 , 13, 14 suggested the most probable mechanism of plasmon catalysis is the intermolecular excitation process (3). It includes a direct transition from the highest occupied molecular orbital (HOMO) to the lowest unoccupied molecular orbital (LUMO) of organic molecules initiated by the excited plasmon resonance exhibiting the same or higher energy than the gap between the HOMO and LUMO of a molecule or molecular adsorbate ${ }^{14,15}$ (Fig. 1A). The most frequent illustration of this mechanism includes the location of Fermi level of plasmonic nanostructure between HOMO and LUMO; however, a more common situation for organic reagents implies HOMO, LUMO levels of energy which are far above or below the Fermi level ${ }^{19,20}$ It means that we have to consider organic molecules with a different structure for the detailed study of plasmon mechanistic aspects. Therefore, here we selected alkoxyamines with different structures to consider their plasmon-induced homolysis due to the simplicity of kinetic measurement using sensitive EPR methods. 


\section{Results and Discussion}

AuNPs- $\mathrm{C}_{4} \mathrm{H}_{9}:$ plasmonic system characterization

Here, we commenced our investigation with the preparation of spherical gold nanoparticles (AuNPs) sized $14.3 \pm 2 \mathrm{~nm}$ modified by lipophilic functional groups (FGs) are prepared via diazonium chemistry (Fig. S1 and Fig. 1B), giving AuNPs- $\mathrm{C}_{4} \mathrm{H}_{9}$. The organic coating prevents the direct interaction between orbitals of gold with the $\pi$-electrons in alkoxyamines structure as back $\pi$-donation causes weakening energy required for $\mathrm{C}-\mathrm{ON}$ bond homolysis. ${ }^{9}$ Moreover, the lipophilic nature of $\mathrm{AuNPs}-\mathrm{C}_{4} \mathrm{H}_{9}$ leads to their stabilization in toluene for further plasmonic experiments with EPR monitoring. The scope of AAs with various substituents in the alkyl and nitroxyl parts (TEMPO-St-NH 2 , SG1-St-NH $2, \mathrm{NH}_{2}$-TEMPO-St, TEMPO-St$\mathrm{COOEtNH}_{2}$, TEMPO-Pyr) (Fig. 1E) was used for the mixing with $\mathrm{AuNPs}-\mathrm{C}_{4} \mathrm{H}_{9}$ in toluene and the further illumination with LED with $660 \mathrm{~nm}$ (Fig. 1D) corresponding to the maximum of plasmon resonance in toluene. The illumination at the plasmon resonance of AuNPs- $\mathrm{C}_{4} \mathrm{H}_{9}$ triggers the $\mathrm{C}-\mathrm{ON}$ bonds homolysis and the subsequent generation of nitroxide, which can be precisely and quickly detected by EPR (Fig. 1B). EPR provides information about the nature and number of radicals leading to careful kinetic measurements.
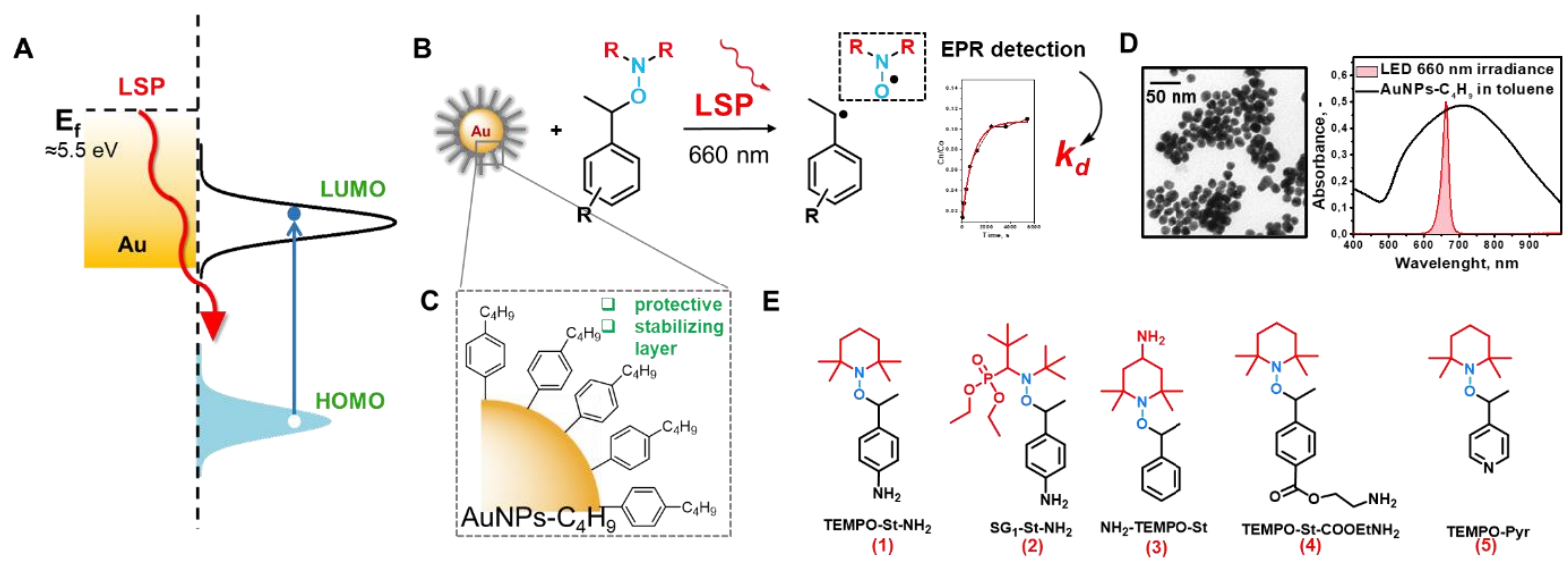

Figure 1. Overview of the experimental strategy. A - Scheme for the direct intramolecular excitation mechanism via localized surface plasmon (LSP) resonance and location of HOMO, LUMO levels, B - 
Scheme of plasmon-induced homolysis of alkoxyamines using AuNPs- $\mathrm{C}_{4} \mathrm{H}_{9}, \mathbf{C}$ - the structure of organic layer, D -TEM image, and UV-Vis spectra of AuNPs- $\mathrm{C}_{4} \mathrm{H}_{9}$, E- structures of alkoxyamines investigated.

Optimization $C(A u N P s) / C(A A)$ ratio and plasmonic homolysis

In order to develop the most reliable system AuNPs- $\mathrm{C}_{4} \mathrm{H}_{9}-\mathrm{AA}$ for the monitoring of plasmon-induced homolysis kinetic, we varied the weight ratio of concentration in AuNPs and alkoxyamines C(AuNPs)/C(AA) from 0.05 to 41 (Fig. S2). The conversion was growing with the C(AuNPs)/C(AA) weight ratio, the ratio 41 was found to be optimal due to the full conversion of AA and high convergence to a linear function. The further increase of $\mathrm{C}(\mathrm{AuNPs}) / \mathrm{C}(\mathrm{AA})$ ratio led to the aggregation (Fig. S2D) of AuNPs- $\mathrm{C}_{4} \mathrm{H}_{9}$ with limiting the optical properties and homogeneity of the probe.

\section{Kinetic study of plasmon-induced AA homolysis}

Using optimal conditions, we performed the plasmon-induced homolysis of $10^{-4} \mathrm{M}$ solutions of TEMPOSt-NH $\mathrm{NH}_{1}$-St-NH $\mathrm{NH}_{2}$-TEMPO-St, TEMPO-St-COOEtNH $\mathrm{NH}_{2}$, TEMPO-Pyr in the presence of AuNPs$\mathrm{C}_{4} \mathrm{H}_{9}$. During the homolysis, we analyzed probes taken after certain periods by EPR analysis, where the intensity of signal corresponds to the number of radical species. Recorded EPR signal (Fig. S3) provide kinetic data (Fig. 2A), where the plasmon induced homolysis was fitted with $1^{\text {st }}$ order kinetic equation. The values of $k_{\mathrm{d}}$ (Fig. 2C) are estimated by fitting the plots of concentration vs. time with equation (1)

$$
\frac{[\text { nitroxide }]_{t}}{[\text { nitroxide }]_{\infty}}=1-\exp ^{-k_{d^{* t}}} \text { eq (1) }
$$




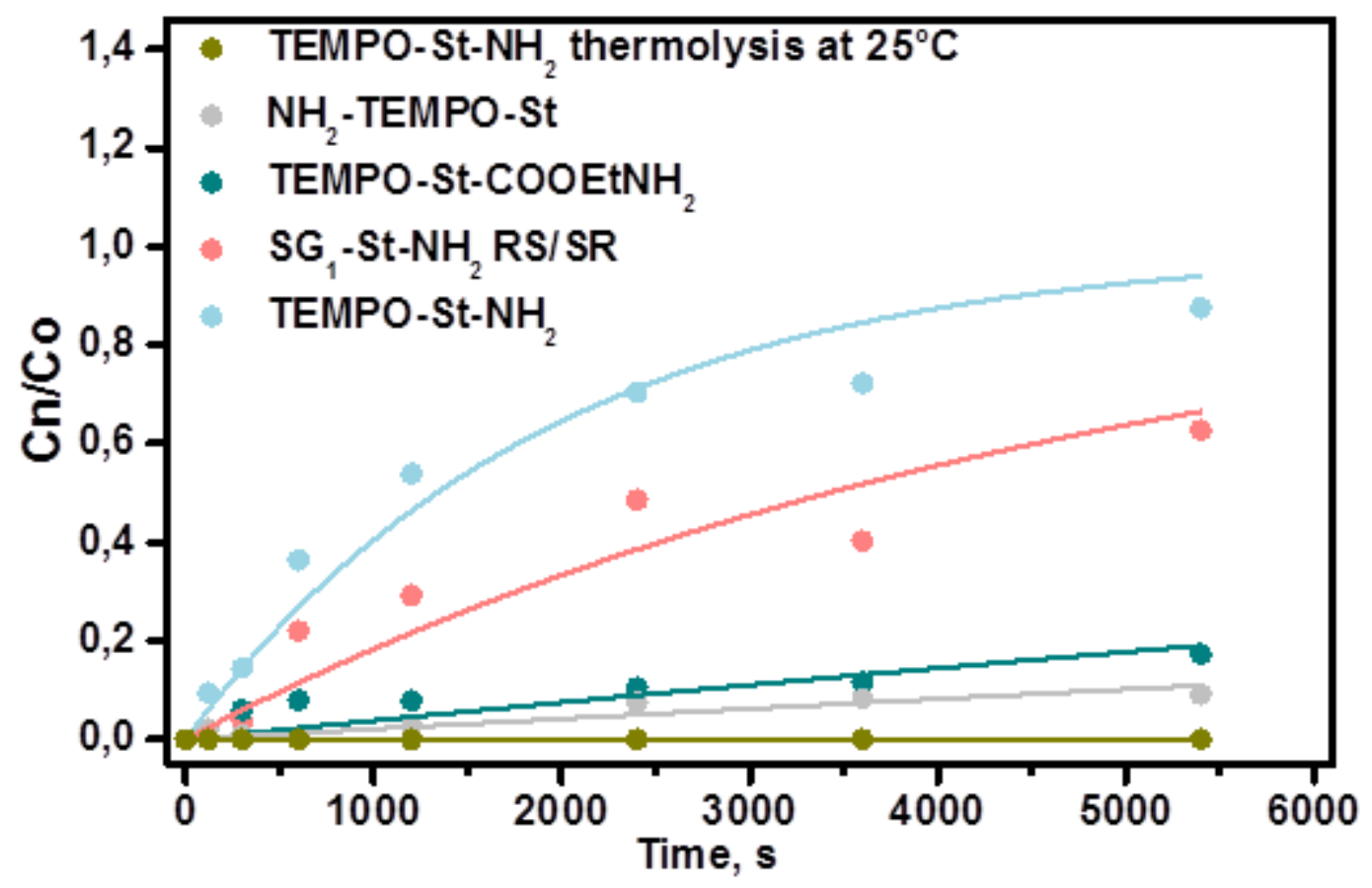

Figure 2. EPR kinetic curves of AAs $(0.1 \mathrm{mM}$ stock solution of appropriate alkoxyamines) in the presence of AuNPs- $\mathrm{C}_{4} \mathrm{H}_{9}$ in weight ratio $\mathrm{C}\left(\right.$ AuNPs- $\left.\mathrm{C}_{4} \mathrm{H}_{9}\right) / \mathrm{C}(\mathrm{AA})=41$ under LED $660 \mathrm{~nm}$ irradiation and thermolysis of TEMPO-St-NH 2 at $25^{\circ} \mathrm{C}$

Table 1. Kinetic data of AAs homolysis of $k^{\prime \prime}{ }_{\mathrm{d}}$ at $100{ }^{\circ} \mathrm{C}, k_{\mathrm{d}}^{\prime}$ at $25^{\circ} \mathrm{C}$, and $k_{\mathrm{d}}$ at $25{ }^{\circ} \mathrm{C}$ under light illumination, activation energies $E_{\mathrm{a}}$, temperature expected $T_{\mathrm{p}}$, and energy of HOMO $E_{\text {Hомо }}$

\begin{tabular}{|c|c|c|c|c|c|c|}
\hline \multirow[t]{2}{*}{ Compound } & $\begin{array}{c}\text { PI- } \\
\text { homolysis }^{a, b}\end{array}$ & $k_{\mathrm{d}}^{\prime}, \mathrm{s}^{-1}$ at $25^{\circ} \mathrm{C}^{\mathrm{c}}$ & $k^{\prime \prime}{ }_{\mathrm{d}}, \mathrm{s}^{-1}$ at 100 & $E_{\mathrm{a}}, \mathrm{kJ} \mathrm{mol}^{-1}$ & $T_{\mathrm{p}},{ }^{\circ} \mathrm{C}^{\mathrm{d}}$ & $E_{\text {номо }}, \mathrm{eV}^{e}$ \\
\hline & $k_{\mathrm{d}}, \mathrm{s}^{-1}$ & & & & & \\
\hline (1)TEMPO-Pyr & $3.5 \cdot 10^{-9 f}$ & $3.5 \cdot 10^{-9}$ & $1.3 \cdot 10^{-4}$ & $130.3^{g}$ & - & -5.72 \\
\hline $\begin{array}{c}\text { (2) } \mathrm{NH}_{2} \text {-TEMPO- } \\
\mathrm{St}\end{array}$ & $2.1 \cdot 10^{-5}$ & $8.8 \cdot 10^{-10}$ & $4.5 \cdot 10^{-5}$ & 133.7 & 95 & -5.25 \\
\hline
\end{tabular}




\section{(3)TEMPO-}

$\mathrm{COOEtNH}_{2}$
$3.9 \cdot 10^{-5}$
$3.2 \cdot 10^{-9}$
$1.3 \cdot 10^{-4}$
130.5
91
$-5.38$

(4)SG1-St-NH

$$
2.0 \cdot 10^{-4}
$$

$1.7 \cdot 10^{-8}$

$4.9 \cdot 10^{-4}$

$124.5^{h}$

93

$-5.18$

(5)TEMPO-St-

$\mathrm{NH}_{2}$

$$
5.2 \cdot 10^{-4}
$$

$1.5 \cdot 10^{-8}$

$4.4 \cdot 10^{-4}$

$127.3^{\mathrm{g}}$

103

$-5.05$

${ }^{a}$ Plasmon-induced homolysis was performed at room temperature in toluene in ratio $\mathrm{C}\left(\mathbf{A u N P s}-\mathbf{C}_{\mathbf{4}} \mathbf{H}_{\mathbf{9}}\right) / \mathrm{C}($ alkoxyamine $)=41$.

${ }^{b}$ Rate constant $k_{\mathrm{d}}$ was calculated from eq. $1 .{ }^{c} \boldsymbol{k}_{\boldsymbol{d}}$ values were calculated from eq. $2 .{ }^{d}$ Estimated $T \mathrm{p}$ assuming a pure thermal effect for the plasmon effect as given in eq. $3 .{ }^{e}$ HOMO energy were estimated from CVA (See FigS4). ${ }^{f}$ In the worse case, plasmon effect cannot be differentiate from the thermal effect. As no homolysis was observed, thermal value of $k^{\prime}$ d at $25{ }^{\circ} \mathrm{C}$ was used. ${ }^{g}$ See ref. 21. ${ }^{g}$ See ref. $9 .{ }^{h}$ See ref. 22.

$$
k_{d}^{\prime} \text { or } k_{d}^{\prime \prime}=2.4 \cdot 10^{14} \cdot \exp \left(\frac{-E_{\mathrm{a}}}{R \cdot T}\right)
$$

During $6000 \mathrm{~s}$ illumination of TEMPO-St-NH 2, SG1-St-NH 2 , with AuNPs- $\mathrm{C}_{4} \mathrm{H}_{9}$ at $660 \mathrm{~nm}$, we noted fast growth of radical concentration in the first $\approx 500 \mathrm{~s}(1)$, and a plateau of nitroxide concentration was reached in less than $3000 \mathrm{~s}$. The $k_{\mathrm{d}}$ values of TEMPO-St-NH $\mathrm{N}_{2}$ and SG1-St-NH were measured to be $5.2 \cdot 10^{-4} \mathrm{~s}^{-1}$ and $2 \cdot 10^{-4} \mathrm{~s}^{-1}$, respectively, exhibiting a 3.6 times difference, while thermal homolysis at $100{ }^{\circ} \mathrm{C}$ for these AA occurs at approximately the same rate $\left(4.4 \cdot 10^{-4} \mathrm{~s}-1\right.$ and $\left.4.9 \cdot 10^{-4} \mathrm{~s}^{-1}\right)$. The most active TEMPOSt- $\mathrm{NH}_{2}$ was homolyzed $10^{4}$ faster under plasmon excitation on $\mathrm{AuNPs}-\mathrm{C}_{4} \mathrm{H}_{9}$ than at room temperature. While for $\mathrm{NH}_{2}$-TEMPO-St, TEMPO-St-COOEtNH $\mathrm{H}_{2}$ the plasmon-induced homolysis occurred one order of magnitude slower, with $k_{\mathrm{d}} 3.9 \cdot 10^{-5} \mathrm{~s}^{-1}$ and $2.1 \cdot 10^{-5} \mathrm{~s}^{-1}$, respectively, than for TEMPO-Sty-NH2 and SG1Sty- $\mathrm{NH}_{2}$. In sharp contrast to TEMPO-sty- $\mathrm{NH}_{2}$ and SG1-sty- $\mathrm{NH}_{2}$, the thermal-derived kinetic constants $k^{\prime \prime}{ }_{\mathrm{d}}$ at $100{ }^{\circ} \mathrm{C}\left(4.5 \cdot 10^{-5}\right.$ and $1.3 \cdot 10^{-4} \mathrm{~s}^{-1}$, respectively) for these AAs differ significantly with $k_{\mathrm{d}}$ (plasmon). In case of TEMPO-Pyr the signal of nitroxide is not detected up to $5000 \mathrm{~s}$ of plasmonic homolysis, while thermal homolysis easily proceeds at $100{ }^{\circ} \mathrm{C}$, i.e., complete conversion of AA into nitroxide. To get a holistic picture, we compared $k_{\mathrm{d}}$ and $k_{\mathrm{d}}$ ' or $k_{\mathrm{d}}$ ' of all AAs obtained from plasmon and thermal homolysis, 
respectively, and did not find the convergence suggesting some alternative explanation of plasmonic AA homolysis.

Previously, we developed a procedure for the estimation of apparent temperature $T_{\mathrm{p}}$ as a descriptor of temperature that can "provide" plasmon at normal conditions using $E_{\mathrm{a}}$ values" according to equation (2) (Fig. 2D). Briefly, $E_{\mathrm{a}}$ was calculated using $k_{\mathrm{d}}$ (thermal homolysis, see SI) and averaged factor $A^{23}$ while $T_{\mathrm{p}}$ was calculated as given by eq. 3 using $E_{\mathrm{a}}$ and $k_{\mathrm{d}}$ (plasmon).

$$
T_{p}=\frac{E_{a}}{R \cdot \ln \frac{2.4 \cdot 10^{14}}{k_{d}(\text { plasmon })}}
$$

The values of $T_{\mathrm{p}}$ for five different AAs were not correlated with $k_{\mathrm{d}}, k_{\mathrm{d}}{ }_{\mathrm{d}}$ and $k{ }^{\prime \prime}{ }_{\mathrm{d}}$ and varied from 91 to $103{ }^{\circ} \mathrm{C}$. Additional, the absence of trends between $k_{\mathrm{d}}$ and $k{ }^{\prime}{ }_{\mathrm{d}}$ (Fig. $\left.4 \mathrm{~A}\right)$ leads us to discard the occurrence of thermal effect and a straightforward correlation of the plasmon-triggered homolysis event with the factors governing the thermal homolysis. These results one more time challenges the key role of plasmonic heating and provokes us to consider chemical factors. For example, the polar, steric, and stabilization effects, ${ }^{24,22}$ and hydrogen bonds ${ }^{25}$ were previously considered as affecting kinetic factors, while effects from all these factors can be merged in the chemical and electronic structure of AA (different location of HOMO and LUMO levels). Therefore, we experimentally measured HOMO and LUMO energies using cyclic voltammetry and UV-Vis data (Fig. S4 and Table S4.1). We attempted to find correlations with energy gap ( $\left.E_{\mathrm{gap}}\right), \mathrm{HOMO}$ and LUMO levels, $E_{\mathrm{HOMO}}$ and $E_{\mathrm{LUMO}}$, respectively (Figure $\left.3 \mathrm{~B}-\mathrm{D}\right)$. The trend of $k_{\mathrm{d}}$ which increases from 1 to 5 is not observed for $k^{\prime \prime}{ }_{\text {d, }} E_{\text {gap }}$ and $E_{\mathrm{LUMO}}$ (Figure 3), whereas it is observed for $E_{\mathrm{HOMO}}$ On the other hand, a good linear dependence with $\mathrm{R}^{2}=0.97$ is observed for the trend of $k_{\mathrm{d}}$ and $E_{\mathrm{HOMO}}$ which is confirmed by correlation between $\log k_{\mathrm{d}}$ Vs $E_{\mathrm{HOMO}}$ (Figure 3D). 
A

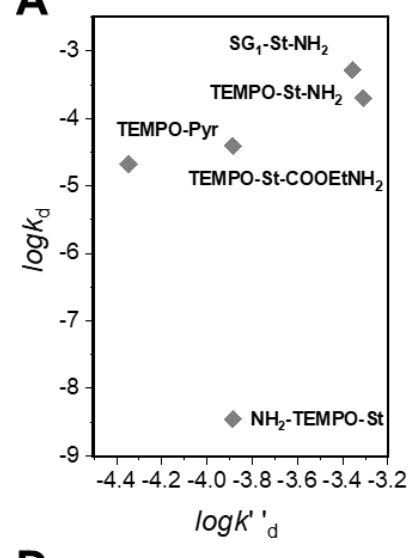

D

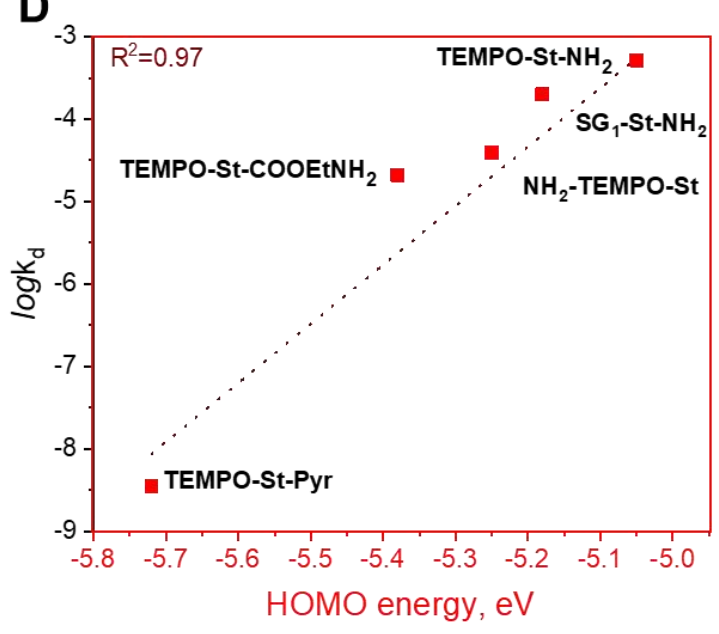

B

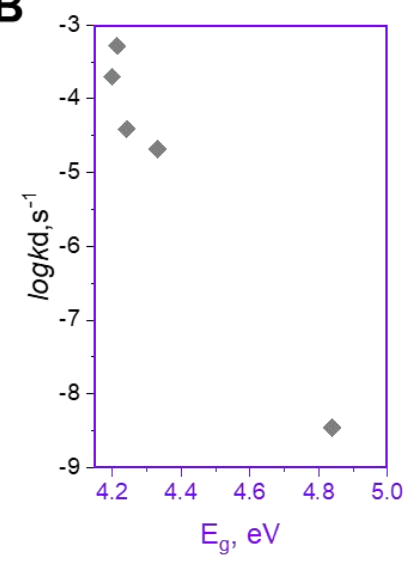

$\mathbf{E}$
C

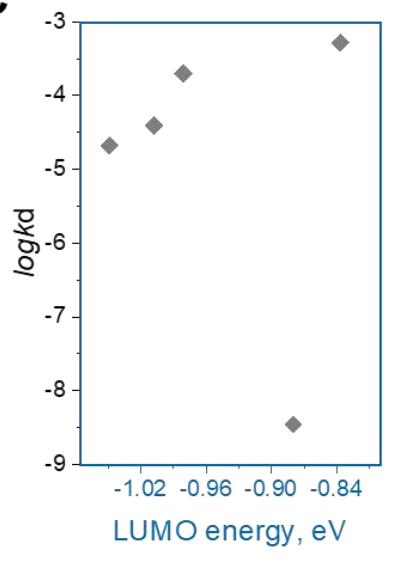

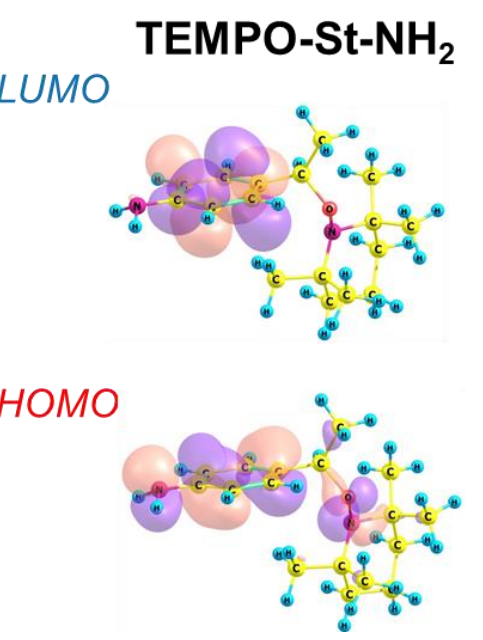

Figure 3 Trends of $\log k_{\mathrm{d}}$ (plasmon) vs $\mathbf{A}-\log k^{\prime \prime}{ }_{\mathrm{d}}\left(\right.$ at $\left.100{ }^{\circ} \mathrm{C}\right), \mathbf{B}-E_{\mathrm{gap}}, \mathbf{C}-E_{\mathrm{LUMO}}, \mathbf{D}-E_{\mathrm{HOMO}}$, and $\mathbf{E}-$ Contour plots for the HOMO and LUMO molecular orbitals for TEMPO-St- $\mathrm{NH}_{2}$

Challenging three mechanisms of plasmon catalysis for AAs homolysis

The changes in $k_{\mathrm{d}}$ and $T_{\mathrm{p}}$ depending on the structures of AA as well as the different trends observed led us to reconsider carefully the three common mechanisms of plasmon catalysis invoked to describe the reactivity observed (Figure 4), namely, plasmonic heating (Figure 4.1), hot electrons (Figure 4.2) and direct intramolecular excitation (Figure 4.3).

The "plasmonic heating" is one of the most frequent mechanism ${ }^{26,27}$ however, the non-correlation between $k_{\mathrm{d}}$ and $k^{\prime \prime}{ }_{\mathrm{d}}$, (Fig. 4A) and difference in $T_{\mathrm{p}}$ clearly highlight a minor occurrence of process (1) in 
the homolysis of the $\mathrm{C}-\mathrm{ON}$ bond. The temperature of the experimental system can be decomposed into macroscopic collective effects, measurable with a thermocamera, and local overheating of nanoparticles, calculated theoretically (Fig. S6). Performed calculations using Generalized Multiparticle Mie theory revealed that under our experimental conditions, local heating in the vicinity of AuNPs is on the order of $10^{-3}{ }^{\circ} \mathrm{C}$ (Fig. S5A, B). Measurements of temperature during plasmon induced homolysis were performed in the reaction vessel with AuNPs- $\mathrm{C}_{4} \mathrm{H}_{9}+\mathrm{TEMPO}-\mathrm{St}-\mathrm{NH}_{2}$ in toluene under $660 \mathrm{~nm}$ LED illumination and thermal camera FLIR ETS320. After $6000 \mathrm{~s}$ illumination, the increase of the reaction temperature in the middle of reaction vessel was found to be $6 \pm 3{ }^{\circ} \mathrm{C}$ (Fig. S6C, D). Taking into account homolysis temperatures for all AAs is larger than $60{ }^{\circ} \mathrm{C}^{9,21,22}$ such change in temperature is considered to be insignificant. Moreover, the increase in temperature of AuNPs- $\mathrm{C}_{4} \mathrm{H}_{9}+\mathrm{TEMPO}-\mathrm{St}_{-} \mathrm{NH}_{2}$ under illumination was found to be linear $\left(\mathrm{R}^{2}=0.96\right)$ (Fig. S6C): light absorption is proportional to the optical power impinging onto the sample. ${ }^{28}$ The case of a photothermal process is, however, different and should not feature such a linear dependence. ${ }^{28}$ To get close mechanistic inside-the variation of LED power from $0-409 \mathrm{~mW} / \mathrm{cm}^{2}$ for the same system demonstrates highly super linear dependence with $\mathrm{R}^{2}$ lin $=0.99$ (Fig. S6), leading us to discard such effect. Indeed, closer fitting of power dependence to the linear rather than exponential increase is a signature of the absence of photothermal effects. However, the maximum LED power $409 \mathrm{~mW} / \mathrm{cm}^{2}$ lead to the deviation toward a super-linear dependence probably to previously described multiphoton absorption. ${ }^{29}$ If to consider the maximum power, coefficient $R^{2}$ lin and $R^{2}$ Arr become 0.74 and 0.73 , respectively, suggesting low convergence with each function.

In the hot electron excitation mechanism (2), electrons with a specific energy are resonantly transferred from metal to the LUMO states resulting in the formation of anionic species, which have not been detected by EPR spectroscopy. This scenario is less probable for plasmon-induced AAs homolysis because the transfer is occurring between Au and LUMO level while $k_{\mathrm{d}}$ of homolysis does not depend on $\mathrm{E}_{\mathrm{LUM}}$. In case of electron transfer, the formation of radical anion of AA is expected and one of the mesolysis pathway affords TEMPO and benzylic-type anion (Fig. S7). The reaction pathway was studied via GCMS 
in the presence of non-degassed Methanol- $d_{4}$ as scavenger of anions. However, the expected 4-(ethyl-1d)aniline was not observed highlighting the absence of anionic species, which excludes mesolysis process. The experimental results is one more argument against the occurrence of hot electrons mechanism in the plasmon-triggered decomposition of AA.
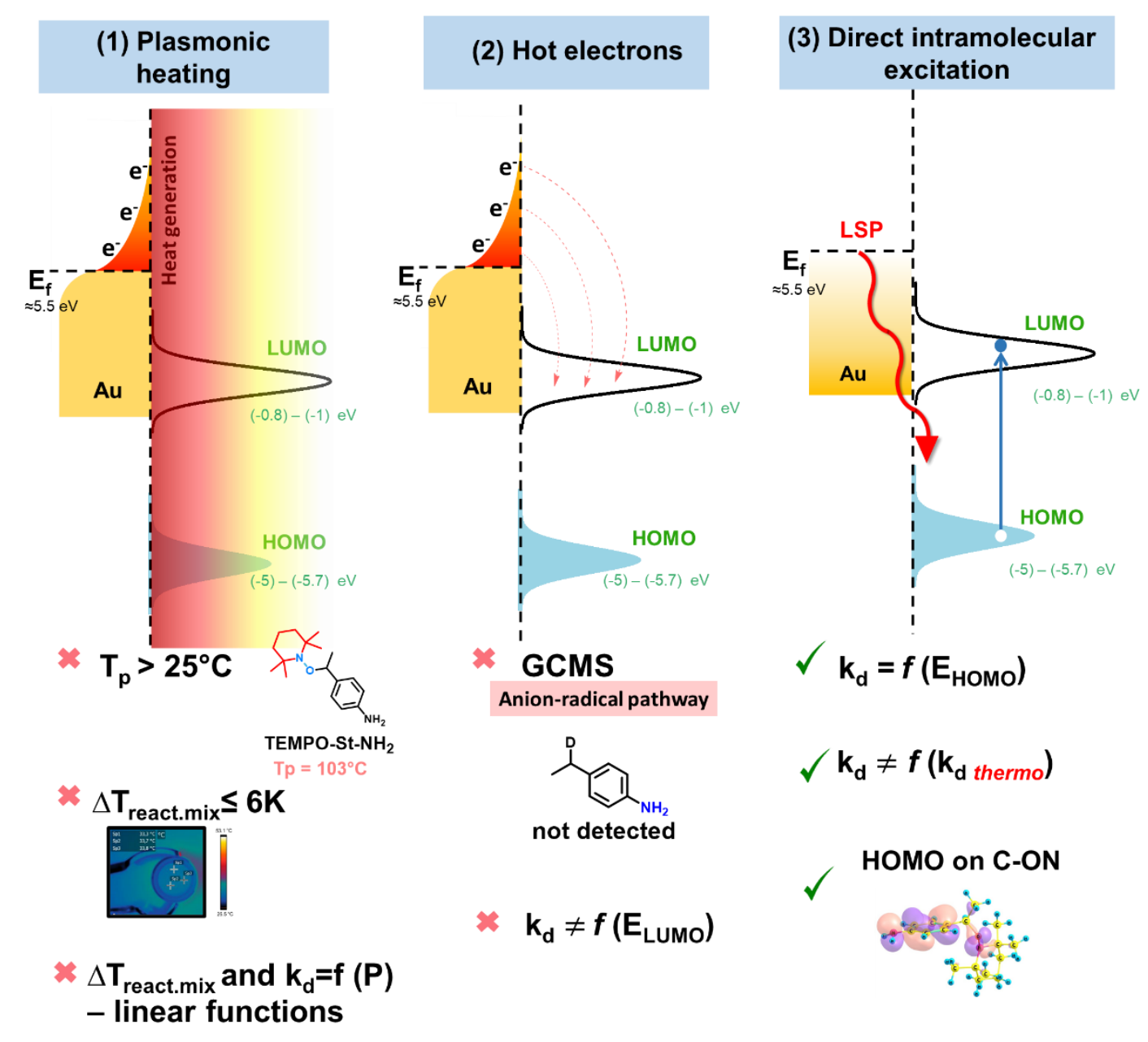

Figure 4. Overview of three possible mechanisms and arguments for/against each of mechanisms: 1) plasmonic heating (2) transfer of a «hot» carriers to the organic molecule, followed by the formation and relaxation of the excited state; (3) intramolecular excitation of an electron to the LUMO via the decay SP.

Finally, the mechanism (3) of intramolecular excitation is the sole, which includes the interaction of electronic orbital HOMO with optically excited surface plasmon. The dependence of the $k_{\mathrm{d}}$ (plasmon) values on the HOMO level suggests that plasmon-induced homolysis of alkoxyamines occurs according to 
the mechanism of intramolecular excitation (Fig. 4). We also calculated the localization of HOMO and LUMO over the AA molecules (Table S4.2, Fig. S3E): only HOMO electron densities are localized on the nitroxyl part of all AA while LUMO is not (contour plots in Tab S4.2) suggesting the critical role of HOMO orbitals in plasmonic homolysis. In our case, in the proximity of the AuNPs- $\mathrm{C}_{4} \mathrm{H}_{9}$ surface, the electronic structure and bond energies in the AAs can be altered significantly with the formation of AuNPs-AA conjugate. Therefore, we investigate AuNPs- $\mathrm{C}_{4} \mathrm{H}_{9}$-AA conjugates using UV-Vis spectroscopy. The excitation of these conjugates may play a crucial role to account for the difference in homolysis rate $k_{\mathrm{d}}$ reported in Table 1 . However, we have to notice here, that AuNPs are coated by $1.8 \mathrm{~nm}$ thick polyphenylene layer, which serve as protecting against any direct chemical interactions with gold surface. Moreover, the usage of toluene solvent prevents the hydrophobic-hydrophobic interactions between AuNPs- $\mathrm{C}_{4} \mathrm{H}_{9}$ and AA. AuNPs- $\mathrm{C}_{4} \mathrm{H}_{9}$ in toluene were mixed with AAs solution, left for interaction and UV-Vis spectra were recorded. In case of all AAs, we observed the shift of the maximum of plasmon resonance of AuNPs- $\mathrm{C}_{4} \mathrm{H}_{9}$ (Fig. 2A, S8) for all AA. Further AuNPs-C $4 \mathbf{H}_{9}+$ AAs are excited using 660 nm LED (denoted in Fig. S8A), where there is almost equal absorbance for all AAs (Fig. S8B). Obtained data suggest that the difference in the homolysis rate is not because of different interaction with AuNPs- $\mathrm{C}_{4} \mathrm{H}_{9}$ but electronic structure of AA. Summing up, clear dependence $k_{\mathrm{d}}$ (plasmon)=f (HOMO) not from LUMO, localization of electron density of $\mathrm{HOMO}$ on $\mathrm{C}-\mathrm{O}$ bonds and correlation of electronic structures of AAs with plasmonic AuNPs- $\mathbf{C}_{4} \mathbf{H}_{9}$ and $k_{\mathrm{d}}$ are convincing that the key mechanism of intramolecular excitation for plasmon-induced AA homolysis (Fig. 4.3). One can wonder if the energy of plasmon excitation is enough to get $100 \%$ conversion of AA according to mechanism (4.3) (presented in Figure 4.3), as the energy gap for TEMPO-St-NH $\mathrm{N}_{2}$ is $\approx 5 \mathrm{eV}$, while $660 \mathrm{~nm}$ light corresponds to the $1.8 \mathrm{eV}$. The dependences of the reaction rate on the incident optical power offer the multiphoton adsorption/ two-stage absorption may occur at $409 \mathrm{~mW} / \mathrm{cm}^{2}$ (Fig. S6) and provide energy multiplication to overcome the energy gap of AAs ${ }^{28}$.

\section{Conclusion}


In summary, we used alkoxyamines as a plasmonic probe (due to one component reaction with $1^{\text {st }}$ order kinetics) mixed with AuNPs coated by protective/stabilizing layer for the study of plasmon induced homolysis under plasmon excitation using EPR monitoring. The detailed kinetic study with optimal experimental conditions revealed the differences in kinetic parameters of homolysis $k_{\mathrm{d}}$ (plasmon) and $k^{\prime \prime}{ }_{\mathrm{d}}$ (thermal) and $T_{\mathrm{p}}$ of different AAs, which cannot be explained by the activation energies of AAs and one more time challenge the key role of plasmonic heating and provoke us consider chemical factors. All investigations - kinetics by EPR, SERS and UV spectroscopies for adsorption, TDFTD for orbitals calculations, cyclic voltammetry for determination of HOMO-LUMO energies, GC-MS for product analysis - concur at promoting the direct intramolecular excitation mechanism (3) to account the triggeredplasmon catalytic homolysis of AA at room temperature and to discard the occurrence of plasmonic heating and hot electron transfer events. Therefore, amongst the three main mechanisms of plasmon catalysis, we discard both the plasmonic heating (process (1)) due to $k_{\mathrm{d}} \neq \mathrm{f}\left(k^{\prime}{ }_{\mathrm{d}}\right)$, to the absence of heating and to the power variation experiments, and the electron transfer (process (2)) due absence of anionic species and to the LUMO energy involvements and to the absence of anion radical products (not detected by GC-MS). Dependence of $k_{\mathrm{d}}$ (plasmon) on the HOMO energy, localization of electron density of HOMO mainly are convincing the key mechanism of intramolecular excitation for plasmon-induced AA homolysis. Our study supports the importance of the chemical and electronic structure of reagents for the plasmon-induced catalysis. We believed that these findings would convince plasmonic chemists to consider photochemical reaction from different points of views - physical as well as chemical.

\section{Methods}

\section{General procedure for kinetic measurements}

A suspension of AuNPs- $\mathrm{C}_{4} \mathrm{H}_{9}$ and solution of alkoxyamine in toluene $0.01 \mathrm{mM}$ were mixed with ratio $\mathrm{C}\left(\mathrm{AuNPs}-\mathrm{C}_{4} \mathrm{H}_{9}\right) / \mathrm{C}(\mathrm{AA}) 40$ to 1 in an NMR tube, treated with ultrasound, and irradiated with a LED $\left(660 \mathrm{~nm}, 409 \mathrm{~mW} / \mathrm{cm}^{2}\right.$; Thorlabs) from bottom to top. LED was closed to the tube without any distance. For kinetic measurements, the NMR tube was removed from the irradiation source and placed into an ESR 
cavity thermostated at $25^{\circ} \mathrm{C}$. After ESR spectra were recorded, and the NMR tube was removed from the ESR cavity and again irradiated by the LED under the same conditions. Sampling was made several times in various periods to plot kinetic curve.

\section{Acknowledgements}

This work was supported by Tomsk Polytechnic University VIU-RSCABS-194/2020 (the kinetic evaluation of homolysis and preparation of NPs) and Russian Science Foundation (№ 20-73-00236 for the preparation of alkoxyamines). R. R. V. thanks the Academy of Finland (projects 1325369, 1315600)

\section{Competing interests}

The authors declare no competing interests.

\section{References}

1. Carter, P., Martirez, J. M. P., Bao, J. L. \& Carter, E. A. First-Principles Insights into PlasmonInduced Catalysis. 1-21 (2020).

2. Zhang, Z., Zhang, C., Zheng, H. \& Xu, H. Plasmon-Driven Catalysis on Molecules and Nanomaterials. Acc. Chem. Res. 52, 2506-2515 (2019).

3. Adleman, J. R., Boyd, D. A., Goodwin, D. G. \& Psaltis, D. Heterogenous catalysis mediated by plasmon heating. Nano Lett. 9, 4417-4423 (2009).

4. Hou, W. \& Cronin, S. B. A review of surface plasmon resonance-enhanced photocatalysis. Adv. Funct. Mater. 23, 1612-1619 (2013).

5. Gellé, A. et al. Applications of Plasmon-Enhanced Nanocatalysis to Organic Transformations. Chemical Reviews vol. 120 986-1041 (2020).

6. Erzina, M. et al. Plasmon-Assisted Transfer Hydrogenation: Kinetic Control of Reaction 
Chemoselectivity through a Light Illumination Mode. J. Phys. Chem. C 125, 10318-10325 (2021).

7. Miliutina, E. et al. Can Plasmon Change Reaction Path? Decomposition of Unsymmetrical Iodonium Salts as an Organic Probe. J. Phys. Chem. Lett. 11, 5770-5776 (2020).

8. Zhang, X. et al. Plasmon-Enhanced Catalysis: Distinguishing Thermal and Nonthermal Effects. Nano Lett. 18, 1714-1723 (2018).

9. Guselnikova, O. et al. Establishing plasmon contribution to chemical reactions: alkoxyamines as a thermal probe. Chem. Sci. 12, 4154-4161 (2021).

10. Guselnikova, O. et al. Plasmon-assisted click chemistry at low temperature: an inverse temperature effect on the reaction rate. Chem. Sci. 12, 5591-5598 (2021).

11. Sytwu, K., Vadai, M. \& Dionne, J. A. Bimetallic nanostructures: combining plasmonic and catalytic metals for photocatalysis. Adv. Phys. X 4, (2019).

12. Nazemi, M., Panikkanvalappil, S. R., Liao, C.-K., Mahmoud, M. A. \& El-Sayed, M. A. Role of Femtosecond Pulsed Laser-Induced Atomic Redistribution in Bimetallic Au-Pd Nanorods on Optoelectronic and Catalytic Properties. ACS Nano 15, 10241-10252 (2021).

13. Kazuma, E. \& Kim, Y. Mechanistic Studies of Plasmon Chemistry on Metal Catalysts. Angew. Chemie Int. Ed. 58, 4800-4808 (2019).

14. Kazuma, E., Jung, J., Ueba, H., Trenary, M. \& Kim, Y. Plasmon-induced chemical reaction of a single molecule. Science (80-. ). 526, 521-526 (2018).

15. Kookhaee, H., Tesema, T. E. \& Habteyes, T. G. Switching a Plasmon-Driven Reaction Mechanism from Charge Transfer to Adsorbate Electronic Excitation Using Surface Ligands. J. Phys. Chem. C 124, 22711-22720 (2020).

16. Kazuma, E., Jung, J., Ueba, H., Trenary, M. \& Kim, Y. Direct Pathway to Molecular 
Photodissociation on Metal Surfaces Using Visible Light. J. Am. Chem. Soc. 139, 3115-3121 (2017).

17. Mo, J. et al. Atomic-Precision Tailoring of Au-Ag Core-Shell Composite Nanoparticles for Direct Electrochemical-Plasmonic Hydrogen Evolution in Water Splitting. Adv. Funct. Mater. 2102517, $1-11(2021)$.

18. Guselnikova, O. et al. Unprecedented plasmon-induced nitroxide-mediated polymerization (PINMP): A method for preparation of functional surfaces. J. Mater. Chem. A 7, 12414-12419 (2019).

19. Vega-Peñaloza, A., Mateos, J., Companyó, X., Escudero-Casao, M. \& Dell'Amico, L. A Rational Approach to Organo-Photocatalysis: Novel Designs and Structure-Property Relationships. Angew. Chemie - Int. Ed. 60, 1082-1097 (2021).

20. Fraser, J. P. et al. Application of a 2D Molybdenum Telluride in SERS Detection of Biorelevant Molecules. ACS Appl. Mater. Interfaces 12, 47774-47783 (2020).

21. Nkolo, P. et al. C-ON bond homolysis of alkoxyamines: When too high polarity is detrimental. Org. Biomol. Chem. 15, 6167-6176 (2017).

22. Audran, G., Brémond, P., Joly, J. P., Marque, S. R. A. \& Yamasaki, T. C-ON bond homolysis in alkoxyamines. Part 12: The effect of the para-substituent in the 1-phenylethyl fragment. Org. Biomol. Chem. 14, 3574-3583 (2016).

23. Walton, J. C. Analysis of Radicals by EPR. Encyclopedia of Radicals in Chemistry, Biology and Materials (2012). doi:10.1002/9781119953678.rad001.

24. Marque, S., Fischer, H., Baier, E. \& Studer, A. Factors influencing the C-O bond homolysis of alkoxyamines: Effects of H-bonding and polar substituents. J. Org. Chem. 66, 1146-1156 (2001).

25. Audran, G. et al. How intramolecular hydrogen bonding (IHB) controls the C-ON bond homolysis 
in alkoxyamines. Org. Biomol. Chem. 15, 8425-8439 (2017).

26. Dubi, Y., Un, I. W. \& Sivan, Y. Thermal effects - an alternative mechanism for plasmon-assisted photocatalysis. Chem. Sci. 11, 5017-5027 (2020).

27. Sivan, Y., Baraban, J., Un, I. W. \& Dubi, Y. Comment on "Quantifying hot carrier and thermal contributions in plasmonic photocatalysis". Science vol. 364 (2019).

28. Baffou, G., Bordacchini, I., Baldi, A. \& Quidant, R. Simple experimental procedures to distinguish photothermal from hot-carrier processes in plasmonics. Light: Science and Applications vol. 9 2047-7538 (2020).

29. Olsen, T. \& Schiøtz, J. Origin of Power Laws for Reactions at Metal Surfaces Mediated by Hot Electrons. Phys. Rev. Lett. 103, 1-4 (2009). 